\title{
Stochastic Improvement of Cyclic Railway Timetables
}

\section{Leo Kroon, Rommert Dekker, Gabor Maroti, Mathijn Retel Helmrich and Michiel Vromans}

\begin{tabular}{|l|l|}
\hline \multicolumn{2}{|l|}{ ERIM REPORT SERIES RESEARCH IN MANAGEMENT } \\
\hline ERIM Report Series reference number & ERS-2006-067-LIS \\
\hline Publication & December 2006 \\
\hline Number of pages & 25 \\
\hline Persistent paper URL & \\
\hline Email address corresponding author & Ikroon@rsm.nl \\
\hline Address & Erasmus Research Institute of Management (ERIM) \\
& RSM Erasmus University / Erasmus School of Economics \\
& Erasmus Universiteit Rotterdam \\
& P.O.Box 1738 \\
& 3000 DR Rotterdam, The Netherlands \\
& Phone: + 31104081182 \\
& Fax: $\quad+31104089640$ \\
& Email: info@erim.eur.nl \\
& Internet: www.erim.eur.nl \\
\hline
\end{tabular}

Bibliographic data and classifications of all the ERIM reports are also available on the ERIM website: www.erim.eur.nl 


\section{ERASMUS RESEARCH INSTITUTE OF MANAGEMENT}

\section{REPORT SERIES \\ RESEARCH IN MANAGEMENT}

\begin{tabular}{|l|l|}
\hline ABSTRACT AND KEYWORDS \\
\hline Abstract & $\begin{array}{l}\text { Real-time railway operations are subject to stochastic disturbances. However, a railway } \\
\text { timetable is a deterministic plan. Thus a timetable should be designed in such a way that it can } \\
\text { cope with the stochastic disturbances as well as possible. For that purpose, a timetable usually } \\
\text { contains time supplements in several process times and buffer times between pairs of } \\
\text { consecutive trains. This paper describes a Stochastic Optimization Model that can be used to } \\
\text { allocate the time supplements and the buffer times in a given timetable in such a way that the } \\
\text { timetable becomes maximally robust against stochastic disturbances. The Stochastic } \\
\text { Optimization Model was tested on several instances of NS Reizigers, the main operator of } \\
\text { passenger trains in the Netherlands. Moreover, a timetable that was computed by the model was } \\
\text { operated in practice in a timetable experiment on the so-called "Zaanlijn". The results show that } \\
\text { the average delays of trains can often be reduced significantly by applying relatively small } \\
\text { modifications to a given timetable. }\end{array}$ \\
\hline Free Keywords & Railway Timetabling, Stochastic Optimization, Robustness \\
\hline Availability & $\begin{array}{l}\text { The ERIM Report Series is distributed through the following platforms: } \\
\text { Academic Repository at Erasmus University (DEAR), DEAR ERIM Series Portal } \\
\text { Social Science Research Network (SSRN), SSRN ERIM Series Webpage } \\
\text { Research Papers in Economics (REPEC), REPEC ERIM Series Webpage }\end{array}$ \\
\hline Classifications & $\begin{array}{l}\text { The electronic versions of the papers in the ERIM report Series contain bibliographic metadata } \\
\text { by the following classification systems: } \\
\text { Library of Congress Classification, (LCC) LCC Webpage } \\
\text { Journal of Economic Literature, (JEL), JEL Webpage } \\
\text { ACM Computing Classification System CCS Webpage } \\
\text { Inspec Classification scheme (ICS), ICS Webpage }\end{array}$ \\
\hline
\end{tabular}




\title{
Stochastic Improvement of Cyclic Railway Timetables
}

\author{
Leo Kroon ${ }^{1,2}$, Rommert Dekker ${ }^{3}$, Gábor Maróti ${ }^{2 \star}$, \\ Mathijn Retel Helmrich ${ }^{3}$, and Michiel Vromans ${ }^{4}$ \\ 1 NS Reizigers, Department of Logistics \\ P.O. Box 2025, NL-3500 HA, Utrecht, The Netherlands \\ 2 Erasmus University, Rotterdam School of Management \\ P.O. Box 1738, NL-3000 DR, Rotterdam, The Netherlands \\ 3 Erasmus University, Rotterdam School of Economics \\ P.O. Box 1738, NL-3000 DR, Rotterdam, The Netherlands \\ 4 ProRail, Spoorontwikkeling \\ P.O. Box 2038, NL-3500 GA, Utrecht, The Netherlands
}

lkroon@rsm.nl

\begin{abstract}
Real-time railway operations are subject to stochastic disturbances. However, a railway timetable is a deterministic plan. Thus a timetable should be designed in such a way that it can cope with the stochastic disturbances as well as possible. For that purpose, a timetable usually contains time supplements in several process times and buffer times between pairs of consecutive trains. This paper describes a Stochastic Optimization Model that can be used to allocate the time supplements and the buffer times in a given timetable in such a way that the timetable becomes maximally robust against stochastic disturbances. The Stochastic Optimization Model was tested on several instances of NS Reizigers, the main operator of passenger trains in the Netherlands. Moreover, a timetable that was computed by the model was operated in practice in a timetable experiment on the so-called "Zaanlijn". The results show that the average delays of trains can often be reduced significantly by applying relatively small modifications to a given timetable.
\end{abstract}

\section{Introduction}

Punctuality of a railway system is a highly important issue, since punctuality is often considered as one of the key performance indicators of a railway system. This is particularly true for passenger trains. In the Netherlands, punctuality of passenger trains is defined as the percentage of trains that arrive at one of the larger railway stations with a delay of

* This research was partially sponsored by the Future and Emerging Technologies Unit of EC (IST priority 6th FP), under contract no. FP6-021235-2 (ARRIVAL). 
less than 3 minutes. Several other countries use a 5 minute margin, or they measure the punctuality only at the final destinations of the trains.

However, measuring the punctuality in these ways is quite inaccurate: a delay of 6 minutes is as bad as a delay of 20 minutes. Moreover, it may also be detrimental to the punctuality itself, since it suggests that there is no problem as long as a delay is less than 3 (or 5) minutes. However, a delay of 2 minutes at a station reduces the probability of an on time arrival at the next station. Therefore, it may be better to measure the punctuality in terms of the average delays of trains.

Delays of trains occur since real-time railway operations are subject to external stochastic disturbances. The latter are also called primary disturbances. However, the underlying railway timetable is a deterministic plan. In order to cope with the disturbances in the real-time operations, a timetable usually contains time supplements in the process times of the trains and buffer times between pairs of consecutive train movements on the same parts of the infrastructure. By the presence of the time supplements, part of the primary stochastic disturbances can be absorbed without giving rise to delays. Moreover, if delays do occur, then the time supplements also enable their absorption. Buffer times between trains reduce the knock-on effects of delays from one train to another. The latter are also called secondary delays.

Thus, to improve the punctuality of a railway system, it is highly relevant to look for an optimal allocation of the time supplements and the buffer times in the timetable. Not only the total amount of time supplements and buffer times, but also their distribution among the processes in the timetable is relevant. The allocation of running time supplements was described recently by UIC (2000). This subject was also studied by Rudolph (2004), Vromans (2005), and Kroon et al. (2007).

In general, allocating more time supplement to a certain process increases the probability that the process can be carried out within the planned time. Therefore, time supplements may add to the predictability of the realized travel times of the passengers. On the other hand, more time supplements also lead to higher planned travel times. Moreover, time supplements may have a negative effect on the realized travel times. Indeed, each minute of running time supplement in the timetable brings the possibility that it is not needed in the operations, since there are no disturbances. Additionally, more time supplements may require more personnel and rolling stock, hence they are negative for the efficiency of the railway system. As a consequence, the time supplements should be chosen by a careful trade-off between these elements. 
The analysis and the improvement of the punctuality of railway services have been studied by several researchers: various relevant models have been developed to that end. The main examples of these models are (i) simulation models, (ii) Max-Plus models, and (iii) analytical models.

Simulation models of railway processes are described by e.g. Bergmark (1996), Wahlborg (1996), Middelkoop and Bouwman (2000), Hürlimann (2001), and König (2001). Models based on Max-Plus algebra are described by e.g. Goverde (1998), (2005), De Kort (2000), and Soto Y Koelemeijer et al. (2000). Finally, analytical models are described by Schwanhäußer (1974), Weigand (1981), Petersen and Taylor (1982), Wakob (1985), Hallowell and Harker (1998), Higgins and Kozan (1998), Carey (1999), Huisman and Boucherie (2001), and Yuan (2006).

A drawback of these existing models is that they are mainly evaluation models and that, based on these models, optimization of the robustness of a timetable can only be achieved by trial-and-error. That is, the timetable is modified and then the evaluation model is used afterwards to evaluate the effect of the modification. These steps are repeated until an acceptable result has been achieved. This iterative process is sometimes referred to as organic planning. Timetable generation models did not explicitly consider stochasticity in the real-time railway operations so far, see e.g. Hooghiemstra et al. (1999) and Peeters (2003).

In contrast with these existing models, this paper describes a Stochastic Optimization Model, see e.g. Birge and Louveaux (1997) and Klein Haneveld and Van der Vlerk (1998). This model can be used to modify a given cyclic timetable for a number of trains that are operated on a certain common railway infrastructure. At the same time, the model can be used to simulate the timetable under construction by operating a number of realizations of the trains in the timetable. These trains are operated as much as possible according to the modified timetable, but subject to primary stochastic disturbances. The main criterion used to modify the timetable is minimization of the average delay of the trains, but other criteria can be handled as well. This minimization is achieved by re-allocating the time supplements and the buffer times in the timetable.

The Stochastic Optimization Model is an improved version of the model described by Vromans (2005) and Kroon et al. (2007). The latter model is based on the same idea and has the same objective, but it uses a linear time axis per train. This feature makes the inclusion of cyclic processes, such as rolling stock circulations, rather cumbersome. The model described in the current paper is based on a cyclic time axis. This also better facilitates network structures of the railway infrastructure. 
The Stochastic Optimization Model is a symbiosis of a timetabling model and a simulation model. The application of the model to several practical cases, together with the evaluation of the obtained results by simulation and by experiments in practice have shown that the improvement of a given timetable by stochastic optimization may lead to a substantial reduction of the average delay of the trains.

The structure of this paper is as follows. In Section 2, we give a simple example with just two consecutive trips of a single train to illustrate the effect of stochasticity on the optimal timetable. In Section 3, we describe the Stochastic Optimization Model in more detail. Section 4 presents results on the existence of an optimal solution, and on convergence of the solutions of the Stochastic Optimization Model to the optimal solution if the number of realizations increases. In Section 5, we describe several further modeling issues. Section 6 presents computational results based on instances of NS Reizigers, the main operator of passenger trains in the Netherlands. Section 7 describes the results of a practical experiment with an improved timetable on the so-called "Zaanlijn" in the Netherlands. The paper is concluded in Section 8.

\section{Two trips: an analytical approach}

In order to illustrate the idea of optimizing a timetable under uncertainty, we start with a straightforward probabilistic analysis of the timetabling problem for just two consecutive trips of a single train.

Suppose that a train is operated over two identical consecutive trips, where on each trip the train incurs an exponentially distributed disturbance with average $1 / \lambda$. In order to be able to cope with these disturbances, a total running time supplement $S$ is to be allocated to the two trips. The running time supplement allocated to trip $t$ is called $s_{t}$ $(t=1,2)$. The dwelling between the two trips does not have any time supplement. The initial delay by the start of the first trip is 0 .

Then for each $d>0$ the probability $P\left(D_{1} \leq d\right)$ that, by the end of the first trip, the train has a delay $D_{1}$ not exceeding $d$ equals the probability that the disturbance on the first trip does not exceed $d+s_{1}$. Hence it can be expressed as follows.

$$
P\left(D_{1} \leq d\right)=1-\exp ^{-\lambda\left(s_{1}+d\right)}
$$

A somewhat more complex analysis shows that for each $d>0$ the probability $P\left(D_{2} \leq d\right)$ that, by the end of the second trip, the train has a delay $D_{2}$ not exceeding $d$ equals 


$$
P\left(D_{2} \leq d\right)=1-\exp ^{-\lambda\left(s_{2}+d\right)}-\lambda\left(s_{2}+d\right) \exp ^{-\lambda(S+d)}
$$

These results clearly show that the delay of the train on the first trip influences the delay on the second trip. Indeed for each $d>0$ we have $P\left(D_{2} \leq d\right)<P\left(D_{1} \leq d\right)$ if $s_{1}=s_{2}$. This is caused by the fact that the first trip starts with zero delay, and the second trip probably not.

Now the standard mathematical analysis for minimizing the average delay (that is, $(i)$ differentiation with respect to $d$ for determining the probability density functions of $D_{1}$ and $D_{2},(i i)$ integration for determining the average delay, and (iii) differentiation with respect to $s_{1}$ and $s_{2}$ for minimizing the average delay) gives the following results for $s_{1}^{*}$ and $s_{2}^{*}$, the optimal values for the running time supplements on trips 1 and 2 .

$$
s_{1}^{*}=\frac{\ln \left(\left(1+\sqrt{1+4 \exp ^{\lambda S}}\right) / 2\right)}{\lambda} \text { and } s_{2}^{*}=S-s_{1}^{*}
$$

These results show the existence of an optimal timetable, given the intensities of the disturbances. They also show that $s_{1}^{*}>S / 2$ and that $s_{2}^{*}<S / 2$. Note that, if $1 / \lambda>S / \ln 2$, the optimal running time supplements $s_{1}^{*}$ and $s_{2}^{*}$ obtained from (3) satisfy $s_{1}^{*}>S$ and $s_{2}^{*}<0$. In this case, it is more appropriate to set $s_{1}^{*}=S$ and $s_{2}^{*}=0$ if negative running time supplements are not allowed.

Table 1. Optimal vs. proportional allocation of running time supplements for $S=1$

\begin{tabular}{cccccc}
\hline $1 / \lambda$ & $s^{*}$ & $S-s^{*}$ & $D^{*}$ & $D^{p}$ & $\Delta$ \\
\hline 0.60 & 0.63 & 0.37 & 0.72 & 0.73 & $1.68 \%$ \\
0.80 & 0.71 & 0.29 & 1.20 & 1.23 & $2.48 \%$ \\
1.00 & 0.80 & 0.20 & 1.71 & 1.77 & $3.14 \%$ \\
1.20 & 0.89 & 0.11 & 2.24 & 2.32 & $3.67 \%$ \\
1.40 & 0.98 & 0.02 & 2.77 & 2.89 & $4.11 \%$ \\
\hline
\end{tabular}

Table 1 presents results obtained by applying the model with $S=$ 1 and several values for $\lambda$. The columns $D^{*}$ and $D^{p}$ give the average delay for the optimal and the proportional allocation of the running time supplements, respectively. In the proportional allocation, each trip gets a running time supplement of 0.5 . These results show that, for a given 
amount of running time supplement, the difference between the optimal and the proportional allocation, represented in column $\Delta$, increases with the intensity of the disturbances. For just two trips, the differences are small, but for more complicated cases the differences can be larger.

Note that the optimal timetable contains a larger part of the total amount of running time supplement on the first trip. This can be explained as follows: a delay by the end of the first trip is measured by the end of the first trip, but it also increases the probability of a delay by the end of the second trip. Thus, roughly speaking, a delay by the end of the first trip counts twice, whereas a delay by the end of the second trip counts only once. In order to reduce the delays by the end of the first trip, a larger part of the running time supplement must be allocated to the first trip. This non-optimality of a proportional allocation of the running time supplements was pointed out earlier by Vromans (2005) and Kroon et al. (2007), also for more than two trips.

\section{Stochastic Optimization Model}

In this section, we describe the Stochastic Optimization Model that can be used to improve a given cyclic timetable for a number of trains on a common part of the railway infrastructure with respect to the average weighted delay of the trains. The latter is achieved by re-allocating the time supplements and the buffer times in the timetable.

\subsection{Sample Average Approximation Method}

The Stochastic Optimization Model is based on a Sample Average Approximation Method, see Shapiro (2000). That is, let $x$ be the vector of decision variables. This vector includes all planned departure and arrival times for one day, thereby taking into account the fact that all hours of a cyclic timetable are basically identical. Then the timetabling part of the model is described by a system $A x \leq b$ of linear inequalities. The set $K$ of feasible timetables is defined by $K:=\{x \mid A x \leq b\}$.

We assume that the primary disturbances are described by a random vector variable $\Delta$. Let $\delta$ be a realization of $\Delta$. That is, $\delta$ is a disturbance vector which assigns a non-negative disturbance to each trip of a day. Let $D(x, \delta)$ be the average weighted delay of the trains when the timetable $x$ is carried out subject to the primary disturbances in $\delta$. We assume that the expectation $\mathbb{E} D(x, \Delta)$ is finite for each $x \in K$. Then we want to find the minimum 


$$
D^{*}=\min \{\mathbb{E} D(x, \Delta): x \in K\},
$$

where the minimum is attained at $x^{*}$. This problem amounts to minimizing the expected weighted delay of the trains. Note that also other elements could be considered in the objective function.

However, since it is difficult to compute $D^{*}$ and $x^{*}$ directly from (4), we use an approximation. That is, we first select a fixed number of realizations $R$ to be used in the timetable optimization. Then we take a random sample of $R$ vectors $\delta_{1}, \ldots, \delta_{R}$ of primary disturbances, and we optimize the timetable given these primary disturbances. The primary disturbances are assumed to be independent of the timetable. Thus we compute

$$
D_{R}^{*}=\min \left\{\frac{1}{R} \sum_{r=1}^{R} D\left(x, \delta_{r}\right): x \in K\right\},
$$

where the minimum is attained at $x_{R}^{*}$. By taking the number of realizations $R$ large enough, we may hope that $x_{R}^{*}$ and $D_{R}^{*}$ are adequate approximations of $x^{*}$ and $D^{*}$. Therefore, we consider $x_{R}^{*}$ and $D_{R}^{*}$ as solutions to (4). More details on the existence of an optimal solution and on the convergence to an optimal solution are described in Section 4.

\subsection{Model description}

The Stochastic Optimization Model contains a timetabling part for determining the timetable and a simulation part for evaluating the robustness of the timetable under construction. The timetabling part of the model shows some similarity with the well-known Periodic Event Scheduling Model (PESP), see Serafini and Ukovich (1989). Several researchers have studied the application of PESP for cyclic railway timetabling, see e.g. Nachtigall (1996) and Peeters (2003).

Note that in our approach it is not essential that the timetable is cyclic. However, the latter reduces the computation time for solving the model, since a cyclic timetable has less degrees of freedom. Moreover, the timetable of NS Reizigers that formed the basis of our computational experiments is cyclic. Therefore, we assume throughout this paper that the timetable is cyclic with a cycle time of one hour.

The objective of the Stochastic Optimization Model is to find a cyclic timetable with a minimum average weighted delay of the trains. To that end, the model uses an initial cyclic timetable as input and, based on that, it constructs an improved cyclic timetable. In order to evaluate and 
optimize the timetable under construction, $R$ realizations of the timetable are operated subject to a priori selected primary stochastic disturbances. Here each realization covers $H$ consecutive hours of the cyclic timetable. In practice, each realization can be seen as a single day.

The $R$ realizations of the timetable are connected with each other only through the timetable and the objective function of minimizing the average delay of the trains, as is expressed in (5). The objective function is computed over the $R$ realizations and over the processes in the $H$ consecutive hours of each realization.

Consecutive hours of each realization are operated one after another. As a consequence, the delayed trains in hour $h$ of realization $r$ may interact with the trains in hour $h+1$ of realization $r$ if they share the same parts of the infrastructure or if they are connected otherwise, e.g. by a passenger or a rolling stock connection. Crew connections are usually non-cyclic. Therefore such connections are kept out of our model.

Whereas in other papers on automated timetable generation determining the cyclic orders of the trains on the tracks is part of the problem to be solved, we assume in the current paper that the cyclic orders of the trains on the tracks are given and cannot be modified. Indeed, the purpose of the current model is to leave the basic structure of the timetable the same as in the initial timetable and to optimally re-allocate the time supplements and the buffer times in that timetable, so that the resulting timetable is more robust than the initial one.

Moreover, we assume that also in the realizations of the timetable the orders of the trains on the tracks is identical to the orders of the trains on the tracks in the improved timetable (and thus to the orders in the initial timetable). Finally, all connections between trains that are defined in the timetable are maintained in the realizations. In other words, the simulation part of the model does not include traffic control decisions. In fact, given the structure of the model, the latter is basically impossible. Both restrictions are explained in Section 5.1.

\subsection{Notation}

We consider a given cyclic timetable with a cycle time $T$. Usually, $T$ equals 60 minutes. Therefore, we will speak about "hours" instead of "cycles" in the remainder of this paper.

A timetable consists of a number of processes that have to be carried out. For example, trains have to run from one station to another, they

have to dwell for a certain period of time in a station, there has to be a certain headway time between two consecutive trains crossing the same 
part of the infrastructure, two trains have to be split or combined, or they have a passenger or a rolling stock connection, etc. Thus there are processes related to single trains and processes related to pairs of trains. The beginning of a process and the completion of a process are called events. For each event, the corresponding event time is to be planned in the timetable. Given the event times of the events, the process times of the processes equal the differences between the event times of the corresponding events. Event times that correspond to an arrival or a departure of a train are assumed to be integer valued.

Since the timetable is cyclic with a cycle time $T$ of one hour, we only have to consider one hour for the planning part of the model. In each hour, $P$ processes are to be carried out and there are $E$ corresponding events. For each process $p$, the events $b(p)$ and $c(p)$ denote the beginning and completion events of process $p$, respectively. The parameter $m_{p}$ denotes the technically minimum process time of process $p$. The given timetable is described in terms of the planned event times $V_{e}$. These parameters denote the offset of the event times in each hour of the timetable. In the given timetable, process $p$ starts at $V_{b(p)}$ and ends at $V_{c(p)}$. Note that, in a cyclic timetable, we may assume without loss of generality that each process time is shorter than the cycle time $T$.

A process $p$ that completely falls within an hour has $V_{b(p)}<V_{c(p)}$. However, a process $p$ that crosses the end of an hour has $V_{c(p)}<V_{b(p)}$. For example, if the departure time and the running time of a train equal 55 and 17 in a cyclic timetable with cycle time $T=60$, then this train's arrival time equals $12=55+17-60$. To describe such situations, we introduce for each process $p$ a binary input parameter $K_{p}$ that records whether or not the corresponding process crosses the end of the hour. In other words, $K_{p}=1$ if and only if $V_{c(p)}<V_{b(p)}$. The model is not allowed to modify these given cyclic orders of the events in the initial timetable.

In the optimized timetable, the planned event time of event $e$ is denoted by the decision variable $v_{e}$. The decision variable $s_{p}$ denotes the planned time supplement for the process time of process $p$. In order to make sure that the end of the hour does not lead to unwanted restrictions for the planned event times, the planned event times are not restricted to the time interval $[0, T-1]$ : they may take any (integer) value. For example, if the original timetable contains an event time at $t=0$ and the planned event times would be restricted to the interval $[0, T-1]$, then this event time could only move forward in time and not backward. The latter is unwanted, and therefore the event times can basically obtain any value. 
The final event times of the timetable can be obtained by transferring the initially obtained ones back into the time interval $[0, T-1]$.

The timetable is evaluated during its modification by operating $R$ realizations of the timetable subject to a priori selected independent stochastic disturbances. Each realization covers $H$ consecutive hours of the cyclic timetable. Hour $h+1$ of realization $r$ takes place after hour $h$ of realization $r$. The stochastic disturbance of process $p$ in hour $h$ of realization $r$ is denoted by the parameter $\delta_{p, r, h}$ for $p=1, \ldots, P, r=1, \ldots, R$, and $h=1, \ldots, H$. The realized event time of event $e$ in hour $h$ of realization $r$ is denoted by the variable $\tilde{v}_{e, r, h}$. The realized event times of each realization are assumed to occur on a linear time axis. Thus they are not restricted to the time interval $[0, T-1]$

Mainly the delays of the events corresponding to arrivals of trains are evaluated, but also other delays can be taken into account. Arrival events are, by definition, the events whose delays are measured. The set of arrival events is denoted by $E_{a}$. The delay of arrival event $e$ in hour $h$ of realization $r$ is denoted by the decision variable $D_{e, r, h}$. The average weighted delay of all trains is denoted by $D$. Delays are weighted since delays at one location may be more harmful for the passengers than delays at other locations. Certain events, such as a departure of a train, should not start before their corresponding planned event times. Such events are called departure events. The set of departure events is denoted by $E_{d}$.

\subsection{Timetabling part of the model}

Most of the constraints to be satisfied in a cyclic timetabling model can be expressed in terms of the planned event times and the planned process times, see e.g. Peeters (2003). For each process $p$ that may include a variable amount of time supplement $s_{p}$ we get the following relation.

$$
m_{p}+s_{p}=v_{c(p)}-v_{b(p)}+K_{p} \times T \text { for } p=1, \ldots, P .
$$

The left-hand side of this equation describes the planned process time of a process $p$ as the sum of the technically minimum process time $m_{p}$ and the variable time supplement $s_{p}$. The right-hand side describes it as the time difference between the planned completion time and the planned begin time of process $p$, thereby taking into account a possible crossing of the end of the hour.

For certain processes also an upper bound $u_{p}$ on the planned process time may be specified. This results in the following more traditional constraints from the PESP model.

$$
m_{p} \leq v_{c(p)}-v_{b(p)}+K_{p} \times T \leq u_{p} \text { for } p=1, \ldots, P .
$$


Obviously, this constraint could also be enforced by introducing an upper bound of $u_{p}-m_{p}$ on the amount of time supplement $s_{p}$ for process $p$.

Other relevant constraints specify that, at each part of the infrastructure, the time difference between the last and the first planned event time in each hour should not exceed the cycle time $T$ minutes. To that end, let $e_{1}$ be the first planned event in an hour on a certain part of the infrastructure, and let $e_{2}$ be the last planned event in an hour on the same part of the infrastructure, then

$$
0 \leq v_{e_{2}}-v_{e_{1}} \leq T-1
$$

These constraints are important since the event times are not restricted to the time interval $[0, T-1]$, as was explained earlier. These constraints guarantee that the obtained timetable can be transferred back into the time interval $[0, T-1]$ after the optimization.

Next, in order to allocate a certain amount of time supplement to the process times, $Q$ subsets $A_{1}, \ldots, A_{Q}$ of processes are selected. Each subset $A_{q}$ of processes is connected with a certain given amount of time supplement $S_{q}$ to be allocated to the processes in $A_{q}$. Then the following constraints are to be satisfied.

$$
\sum_{p \in A_{q}} s_{p} \leq S_{q} \text { for } q=1, \ldots, Q
$$

For example, such a constraint may indicate that a certain total amount of running time supplement is to be allocated to the consecutive running times along the line of a single train. However, a certain amount of time supplement may also have to be allocated to a number of lines together.

Note that for modeling the processes that involve a pair of trains, the assumption that the orders of the events should remain unchanged, is essential. Indeed, if the orders of the events would not be known a priori, then additional binary variables would be required to model these. This feature would highly complicate the solution process of the model, as is described in Section 5. However, given the orders of the events, all constraints can be described as in (6)-(9).

Finally, non-negativity constraints have to be imposed on the variables $s_{p}$, and if one wants to obtain a timetable that is specified in integer minutes, then integrality constraints have to be imposed on the corresponding event times as well. 


\subsection{Simulation part of the model}

Recall that we assume that in the realizations the orders of the events are the same as in the timetable. Thus, the simulation part of the model does not include traffic control decisions.

As was mentioned earlier, the $H$ hours of each realization are operated one after another. A process $p$ with $K_{p}=0$ has $V_{b(p)}<V_{c(p)}$. Thus it is planned within a single hour. Therefore, we assume that a process $p$ with $K_{p}=0$ always ends in the same hour as the hour it started in. However, a process $p$ with $K_{p}=1$ has $V_{c(p)}<V_{b(p)}$. Obviously, in the realizations it is impossible that a process ends earlier than it started. Therefore we assume that a process $p$ with $K_{p}=1$ always ends in a later hour than the hour it started in. Thus, a process $p$ with $K_{p}=1$ starts in hour $h$ of realization $r$ at $\tilde{v}_{b(p), r, h}$. This process ends at $\tilde{v}_{c(p), r, h+1}$.

The foregoing implies that the constraints linking the event times of the processes to the technically minimum process times and the disturbances are the following.

$$
\begin{aligned}
& m_{p}+\delta_{p, r, h} \leq \tilde{v}_{c(p), r, h+K_{p}}-\tilde{v}_{b(p), r, h} \\
& \quad \text { for } p=1, \ldots, P ; r=1, \ldots, R ; h=1, \ldots, H .
\end{aligned}
$$

As a consequence, a delayed train in hour $h$ of realization $r$ may influence the trains in hour $h+1$ in realization $r$ if one of its related processes crosses the end of the hour. Note that realized process times do not have an upper bound, in contrast with the planned process times that may have an upper bound. Indeed, the realized process times should have the freedom to be extended basically indefinitely, depending on the sizes of the disturbances. Note that (10) is an inequality and not an equality. Indeed, (10) only deals with primary disturbances. However, trains may also pick up secondary delays from interactions with other trains.

Departure events should not occur too early, and a delay corresponds to a late arrival event. This results in the following constraints.

$$
\begin{aligned}
v_{e}+h \times T & \leq \tilde{v}_{e, r, h} \\
& \text { for } e \in E_{d} ; r=1, \ldots, R ; h=1, \ldots, H, \\
\tilde{v}_{e, r, h}-\left(v_{e}+h \times T\right) \leq D_{e, r, h} & \\
& \text { for } e \in E_{a} ; r=1, \ldots, R ; h=1, \ldots, H .
\end{aligned}
$$

Here we use the cyclic character of the timetable, since the planned event time of event $e$ in hour $h$ of realization $r$ equals $v_{e}+h \times T$. Import delays 
of trains that enter the studied area can be modeled similarly. All delay variables $D_{e, r, h}$ are non-negative. Indeed, positive delays of trains should not be compensated by negative delays of other trains.

In the model, the objective is to minimize the average weighted delay of the trains. Thus the objective is to

$$
\text { minimize } D=\sum_{e \in E_{a}} \sum_{r=1}^{R} \sum_{h=1}^{H} w_{e} D_{e, r, h} /\left(\left|E_{a}\right| \times R \times H\right) \text {. }
$$

Here the weights $w_{e}$ indicate the weights of the different delays.

Note that maximizing the punctuality of the trains is much harder than minimizing the average delay of the trains. Indeed, for maximizing the punctuality, one needs for each arrival event a binary decision variable that records whether or not the realized arrival takes place within the punctuality margin. Therefore, if one wants to improve the punctuality, this may be accomplished indirectly by penalizing delays exceeding the punctuality margin more severely than other delays.

\section{Existence and convergence}

As was mentioned already in Section 3.1, the Stochastic Optimization Model is based on a Sample Average Approximation Method. In the following, $x$ denotes the vector of decision variables, and the timetabling part of the model is described by a system $A x \leq b$ of linear inequalities. The set $K:=\{x \mid A x \leq b\}$ of feasible timetables is compact.

The primary disturbances are described by a random vector variable $\Delta$, and $\delta$ is a realization of $\Delta$. Recall that the function $D(x, \delta)$ denotes the average weighted delay of the trains when the timetable $x$ is carried out subject to the primary disturbances $\delta$. We assume that $D(x, \delta)$ is a continuous function of $x$. It is not difficult to verify that this function is convex in $x$ and satisfies $|D(x, \delta)| \leq C \cdot\|\delta\|$ for all $x$ and $\delta$ with some positive constant $C$. The latter depends on the number of trips in each realization. We also assume that the expectation $\mathbb{E} D(x, \Delta)$ is finite for each $x \in K$. These are realistic assumptions. Then we want to find

$$
D^{*}=\min \{\mathbb{E} D(x, \Delta): x \in K\},
$$

where the minimum is attained at $x^{*}$. One easily verifies that $\mathbb{E} D(\cdot, \Delta)$ is a continuous function of $x$ on the compact set $K$. This implies that the minimum $D^{*}$ that we are looking for exists indeed. 
In the Sample Average Approximation Method, we select a fixed number of realizations $R$ to be used in the timetable optimization. Then we take a random sample $\delta_{1}, \ldots, \delta_{R}$ of the primary disturbances that are assumed to occur in the railway operations during these realizations. Finally, we optimize the timetable over these realizations and given these primary disturbances. Thus we compute

$$
D_{R}^{*}=\min \left\{\frac{1}{R} \sum_{r=1}^{R} D\left(x, \delta_{r}\right): x \in K\right\},
$$

where the minimum is attained at $x_{R}^{*}$. Again, the minimum exists.

The convergence properties of $D_{R}^{*}$ and $x_{R}^{*}$ have been studied extensively in recent years, see e.g. King and Rockafellar (1993), Kaniovski et al. (1995), Shapiro (2000), Shapiro and Homen-de-Mello (2000), and Linderoth and Shapiro (2002). In these papers, general results are derived from the Law of Large Numbers.

These results imply that, under the assumptions of our model, the function values $D_{R}^{*}$ tend to $D^{*}$ with probability 1 for increasing values of $R$. Moreover, dist $\left(x_{R}^{*}, K^{*}\right)$ tends to zero with probability 1 for increasing values of $R$. Here $K^{*}:=\left\{x \in K: \mathbb{E} D(x, \Delta)=D^{*}\right\}$. It follows that the Sample Average Approximation Method leads to appropriate approximations of $x^{*}$ and $D^{*}$ for increasing values of $R$.

\section{$5 \quad$ Further modeling issues}

\section{$5.1 \quad$ Traffic control}

The model described in this paper does not include operational traffic control decisions. That is, in each realization of the processes in the timetable, the cyclic orders of the trains on the tracks are the same as in the initial timetable. Moreover, connections between trains that are defined in the timetable are always maintained in the realizations. This may seem to be a restriction of the model. However, given the structure of the Stochastic Optimization Model, it is basically impossible to incorporate operational traffic control decisions into such a model.

Indeed, all disturbances are generated prior to solving the model. Thus, if operational traffic control decisions would be incorporated into the model, then at each point of time these decisions would be taken under complete knowledge of all disturbances in the future. In other words, the traffic control decisions generated by the model could anticipate on certain disturbances in the future. This is not realistic in practice, where, at each 
point of time, one has to decide in the operations based on information that is available at that particular time instant only.

Note that the stochastic optimization model is meant to increase the robustness of the timetable against relatively small disturbances. In fact, a timetable cannot be made robust against large disruptions. Small disturbances usually do not require traffic control decisions. Therefore, the fact that traffic control decisions cannot be incorporated into the model is not really a problem.

\subsection{Variable planned train orders}

The model described in this paper assumes that the planned cyclic orders of the trains on the tracks are given by the input parameters $K_{p}$. If the model would also be allowed to modify the planned cyclic orders of some of the trains on the tracks, then some more flexibility could be built into the model. Note that, also in this case, we have to retain the assumption that in the operations the cyclic orders of the trains on the tracks are the same as in the plan, as was explained in Section 5.1.

First, for each process $p$ corresponding to events that may be switched in order, the former input parameter $K_{p}$ is to be treated as a binary decision variable. The latter also implies that, since train orders are not fixed now, constraints such as headway constrains have to be included for all pairs of trains on a certain location instead of for pairs of consecutive trains only. Also constraints (8) have to be taken into account for all pairs of trains, since in this case it is not known a priori which events are the first and the last planned events on a certain location in each hour. The remaining timetabling part of the model may remain the same.

The simulation part of the model has to be modified as well. For each process $p$ where $K_{p}$ is a decision variable, constraints (10) obviously have to be modified, since such constraints are highly non-linear. These constraints are to be replaced by the following ones.

$$
\begin{array}{r}
l_{p}-K_{p} \times T \leq v_{c(p), r, h}-v_{b(p), r, h} \leq u_{p}+K_{p} \times T \\
\quad \text { for } p=1, \ldots, P ; r=1, \ldots, R ; h=1, \ldots, H \\
l_{p}-\left(1-K_{p}\right) \times T \leq v_{c(p), r, h+1}-v_{b(p), r, h} \leq u_{p}+\left(1-K_{p}\right) \times T \\
\text { for } p=1, \ldots, P ; r=1, \ldots, R ; h=1, \ldots, H
\end{array}
$$

Thus constraints (16) can be active only if $K_{p}=0$. In that case, constraints (17) are void. If $K_{p}=1$, the situation is similar but reversed. Note that these constraints are quite bad for the computation times of the model, due to their Big M-character. 


\subsection{Peak and off-peak hours}

Railway services usually have to deal with non-symmetric peak demand. In the morning peak, many passengers travel towards their work in the large cities. In the afternoon peak, they travel in the reverse direction. In order to accommodate this non-symmetric peak demand in a cyclic timetable, a number of additional trains may be operated in the peak direction during the peak hours. During the off-peak hours, the time slots of these additional trains are empty. Note that in this case the timetable is not completely cyclic anymore.

We included this difference between the timetable in peak and off-peak hours in our model. However, since this extension of the model leads to a rather cumbersome description, we skip the details. The details can be found in Retel Helmrich (2006).

In the timetabling part of the extended model, which still covers only a single hour, we included all trains. That is, we included the regular trains and the trains that are operated only during one of the peak periods. A timetable is feasible in the extended model if $(i)$ the regular trains do not have conflicts with each other, $(i i)$ the morning peak trains fit between the regular trains, and (iii) the afternoon peak trains fit between the regular trains as well. A virtual "conflict" between a morning peak train and an afternoon peak train is neglected by the model.

In the simulation part of the model, we assume that the peak trains are operated only during the appropriate peak hours. Note that this implies that, during different hours of the day, different constraints for the relations between trains are to be taken into account. For example, the headway constraints depend on the actual hour of the day.

\section{Computational results}

In this section we present computational results that were obtained by applying the Stochastic Optimization Model to a case based on the 2007 timetable for the northern part of North-Holland in the Netherlands (the so-called "Kop van Noord-Holland", see Figure 1).

The computational results were obtained by implementing the model in the modeling system OPL Studio 3.7 running on Windows XP. The model was solved by CPLEX 9.0. The hardware was an Intel Pentium 4 processor with a clock speed of $3.0 \mathrm{GHz}$ and 1 GB internal memory.

In the simulation part of the model, we could handle at most 420 hours at the same time. These 420 hours were split into $R=20$ realizations (days) of $H=21$ hours. This lead to a model with $500.000+$ decision 
variables and $400.000+$ constraints. The number of integer variables for representing the planned event times in the timetable is $1.000+$.

It should be noted that Stochastic Optimization Models cannot only be solved by (Mixed Integer) Linear Programming, but also by more dedicated optimization methods. Such methods may utilize the fact that a Stochastic Optimization Model usually consists of a relatively small planning part and a relatively large simulation part. The size of the simulation part strongly depends on the number of realizations. A dedicated method can handle this part more efficiently than (Mixed Integer) Linear Programming. For a dedicated method for solving robust timetabling problems based on Convex Optimization, see Maróti (2006).

\subsection{Case description}

The major part of the infrastructure in the "Kop van Noord-Holland" is double-track. However, north of Schagen (Sgn) the infrastructure is just single-track with double-track sections at the stations Anna Paulowna (Ana) and Den Helder Zuid (Hdrz). Also the connections between Heerhugowaard (Hwd) and Hoorn (Hn) and between Enkhuizen (Ekz) and Hoorn Kersenboogerd (Hnk) are single-track sections with double-track sections at most underway stations.

The 2007 timetable for the "Kop van Noord-Holland" is cyclic with a cycle time of 60 minutes. Almost all train lines are operated there twice per hour with a cycle time of 30 minutes, but there are some exceptions.

The number of passenger trains in this area is about 50 per hour. In addition to the passenger trains, each hour includes about 6 time slots for cargo trains. In the operations, only a subset of these time slots is actually used. A further complicating factor is the fact that the "Kop van Noord-Holland" contains several bridges that must be opened sometimes for ships. Therefore, they are temporarily not available for railway traffic.

The trains that enter the "Kop van Noord-Holland" from outside the area, such as the intercity trains of the 3000 line and the regional trains of the 4000 line, are responsible for a lot of import delays in Amsterdam (Asd). Also in Haarlem (Hlm) and near Amsterdam Sloterdijk (Assh), import delays have to be taken into account.

\subsection{Disturbance distributions}

An important part of the data is a description of the disturbances that are to be taken into account in the simulation part of the model. In the model, we distinguish between import delays, dwell time disturbances 


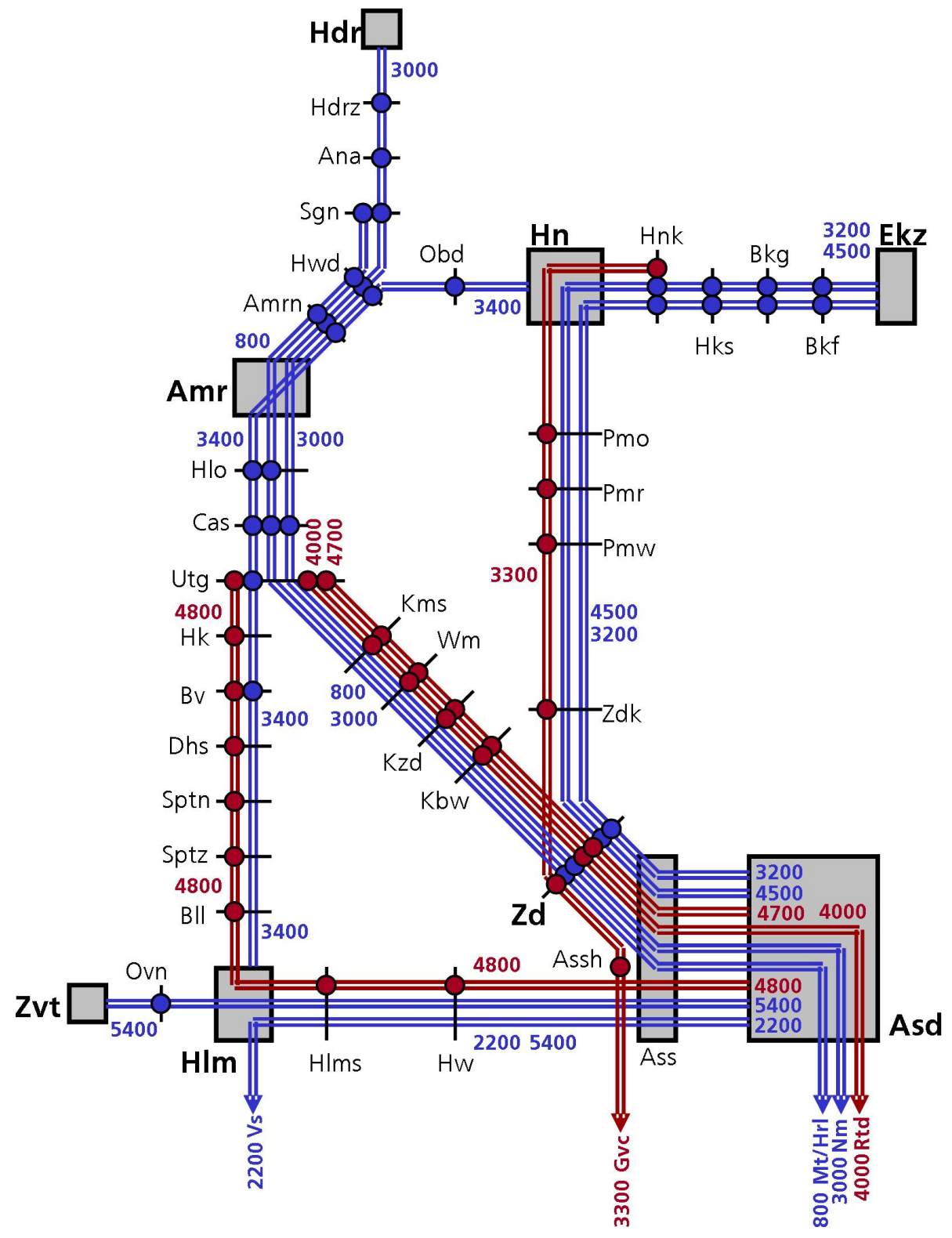

Fig. 1. The train lines in the "Kop van Noord-Holland" in the 2007 timetable. 
and running time disturbances. For determining the intensities of these disturbances, we used an analysis tool developed by NS Reizigers for analyzing log files of realized train movements. These data describe in detail the realized train movements in a certain period of time. We used realization data from weekdays in all four weeks of February 2006. An explanation for using these data is given below.

Note that the realization data describe delays of trains instead of the underlying primary disturbances, e.g. varying dwell times due to varying numbers of passengers or varying running times due to train driver behavior. Delays of trains are the result of a mixture of primary disturbances and secondary disturbances (knock-on delays). However, by filtering the unhindered dwell times and train movements, the analysis tool is able to estimate the distributions of the primary disturbances. Moreover, we assumed that the primary disturbances are independent of the operated timetable. The latter is a realistic assumption, which allows us to use realization data from 2006 for improving the timetable for 2007.

Based on this analysis of the realization data, we determined the intensities (averages) of the primary disturbances. For the underlying distribution of these disturbances, we assumed the exponential distribution. This choice is motivated by a number of studies in this respect, see Schwanhäußer (1974), Ferreira and Higgins (1996), Goverde et al. (2001), and Yuan (2006). For a more detailed description of the data preparation process, we refer to Retel Helmrich (2006).

\subsection{Results}

In the first set of experiments we compared three timetables: $(i)$ the original timetable, (ii) a timetable which was obtained from the Stochastic Optimization Model with the restriction that the running times could change only one minute (referred to as the "restricted SOM timetable"), and (iii) a timetable which was obtained from an optimization without that constraint (referred to as the "unrestricted SOM timetable"). These variants took 33 hours and 16 hours of computation time to arrive at an optimality gap of less than $1.5 \%$. The results are shown in Table 2 .

In this table, the row "Avg. delay $>3$ min." refers to the average of the delays exceeding the 3 minute margin that is taken into account in the punctuality measures. The row "Avg. delay at borders" refers to the delays of trains that leave the studied area and that have a passenger or rolling stock connection with another train at the border. The rows "Punct. (all stations)" and "Punct. (official)" refer to the average punctuality of the trains measured over all stations, and measured in 
the directions where the punctuality is measured officially. The latter are Alkmaar (Amr, from the South and from the North), Amsterdam (from the North), and Haarlem (from the East), see also Figure 1. The high punctuality in Haarlem is due to the fact that not all trains that arrive in Haarlem are taken into account. The objective function is a combination of the penalties that are taken into account in the optimization. The main elements are the average delays of the trains. Delays exceeding the 3 minute margin have a higher weight, and the same holds for delays in Alkmaar, Amsterdam, and Haarlem.

Table 2. Comparison of the original timetable with the SOM timetables

\begin{tabular}{lccc}
\hline & $\begin{array}{c}\text { original } \\
\text { timetable }\end{array}$ & $\begin{array}{c}\text { restricted } \\
\text { SOM timetable }\end{array}$ & $\begin{array}{c}\text { unrestricted } \\
\text { SOM timetable }\end{array}$ \\
\hline Avg. delay (min.) & 1.22 & 0.94 & 0.92 \\
Avg. delay > 3 min. (min.) & 0.29 & 0.18 & 0.16 \\
Avg. delay at borders (min.) & 2.44 & 2.12 & 2.14 \\
Punct. (all stations) & $85.5 \%$ & $90.2 \%$ & $90.9 \%$ \\
Punct. (official) & $86.7 \%$ & $92.4 \%$ & $93.4 \%$ \\
Punct. Alkmaar South & $80.9 \%$ & $90.4 \%$ & $93.8 \%$ \\
Punct. Alkmaar North & $72.3 \%$ & $88.2 \%$ & $92.3 \%$ \\
Punct. Amsterdam North & $87.5 \%$ & $91.2 \%$ & $90.8 \%$ \\
Punct. Haarlem East & $100.0 \%$ & $100.0 \%$ & $100.0 \%$ \\
Objective function & 37,572 & 27,276 & 26,115 \\
\hline
\end{tabular}

Based on the results in Table 2, the following conclusions can be drawn. The Stochastic Optimization Model yields timetables that are more robust than the original one. Indeed, the official punctuality figures show a marked improvement: an increase from $86.7 \%$ to $92.4 \%$ and $93.4 \%$ respectively. The objective function improves by $27 \%$ and $30 \%$ respectively for the SOM timetables. The differences between the restricted and unrestricted timetable are small, but still interesting. Note that the SOM timetables improve the original timetable in all aspects.

\subsection{Sensitivity analysis}

A second set of experiments concerns a sensitivity analysis of the results. The question is to which extent the results depend on the intensities of 
the disturbances. We again compare the two SOM timetables with the original one. However, in this case we evaluate the timetables, without re-optimization of the timetables, according to different disturbance distributions. We scale the averages of the original disturbance distributions with a factor varying from $-30 \%$ to $+30 \%$ with steps of $5 \%$. One could interpret this as e.g. days with different types of weather. For the comparison of the three timetables, we use identical random numbers, but for different averages we did a re-sampling.

Also in these experiments, the SOM timetables performed better than the original timetable. The differences in the objective function increase with the intensities of the disturbances, but the relative differences decrease. For disturbances which are on average 10\% lower than the initially assumed ones, the objective function values are 28,562, 20,263, and 19,739 for the original, restricted, and unrestricted timetable. For disturbances which are on average $10 \%$ higher than the initially assumed ones, these figures are 56,781, 43,313, and 40,539, respectively.

In a final set of experiments, we also re-optimized the two SOM timetables under slightly modified disturbance distributions. In these cases, it turned out that the obtained timetables changed only slightly in comparison with the timetables that were obtained under the initially assumed disturbance distributions. It can be concluded that the results obtained by the Stochastic Optimization Model provide an improvement over the original timetable, and that these results are rather stable under varying intensities of the disturbance distributions.

\section{Timetable experiment in practice}

During the weeks 22 to 29 of 2006 (May 28 until July 23), a timetable generated by the Stochastic Optimization Model was tested in practice on the so-called "Zaanlijn". The "Zaanlijn" is part of the "Kop van NoordHolland": it is the north-south connection between Den Helder (Hdr) and Amsterdam (Asd), see Figure 1. Note that a modified timetable was also operated on the "Zaanlijn" during weeks 30 and 31 of 2006, but this period was not representative due to other changes in the timetable.

The "Zaanlijn" has been notorious for its relatively low punctuality for several years. That is, the punctuality of the "Zaanlijn" was always significantly lower than the overall punctuality over all of the Netherlands. For this reason, it was decided by the top management of NS to carry out a number of experiments by temporarily operating slightly different timetables there. To that end, the Stochastic Optimization Model was 
applied to the 2006 timetable of the "Zaanlijn", more or less in the same way as described in Section 6. In the optimization, the total amount of running time supplement in the timetable remained the same, and the dwell times were unchanged. As a consequence, the individual travel times of the passengers changed only marginally.

\section{Results of the experiment}

Table 3 shows the average punctuality of the trains on the "Zaanlijn" as well as the average overall punctuality in the Netherlands during the weeks 22 to 29 of 2006 of the timetable experiment. This table shows that, during the timetable experiment, the punctuality figures of the "Zaanlijn" are quite comparable with those of the overall punctuality. Note that weeks 24,27 and 29 were weeks with a relatively large number of major disruptions, which had a negative effect on the punctuality.

Table 3. Punctuality of the "Zaanlijn" and the overall punctuality

\begin{tabular}{lccccccccc}
\hline Week & 22 & 23 & 24 & 25 & 26 & 27 & 28 & 29 & Average \\
\hline "Zaanlijn" & $89.7 \%$ & $87.5 \%$ & $80.5 \%$ & $89.5 \%$ & $85.3 \%$ & $74.8 \%$ & $90.2 \%$ & $85.8 \%$ & $85.4 \%$ \\
Overall & $88.6 \%$ & $87.9 \%$ & $82.6 \%$ & $88.8 \%$ & $86.5 \%$ & $75.4 \%$ & $87.3 \%$ & $81.2 \%$ & $84.8 \%$ \\
\hline
\end{tabular}

As a comparison, the average punctuality of the "Zaanlijn" over the first 13 weeks of 2006 was $79.4 \%$, and the average overall punctuality in the Netherlands over this period was $86.5 \%$. Thus the figures in Table 3 suggest that the application of the Stochastic Optimization Model had a positive effect on the punctuality of the "Zaanlijn". Note that, due to the relatively short period of the timetable experiment, it is hard to draw definitive conclusions based on the above figures. For example, the fact that a timetable experiment was carried out may have had a positive effect already on the punctuality figures of the "Zaanlijn".

Anyway, it turned out that the effects of the improved timetable were quite similar to what was expected. That is, at moments that there are only small primary disturbances, the improved timetable is better able to absorb these small disturbances. At moments that there are large disruptions, the improved timetable does not give a clear advantage over the original timetable. Therefore, on average, the improved timetable seems to perform better than the original one. 


\section{Conclusions and further research}

In this paper we described a Stochastic Optimization Model for improving the robustness of a given cyclic railway timetable. The model is an improved version of the model described by Vromans (2005) and Kroon et al. (2007). An improvement of the current version of the model is that it is based on a cyclic time axis, which better facilitates a network structure of the railway infrastructure as well as the inclusion of cyclic relations in the model, e.g. related to rolling stock circulations.

This paper also addresses existence and convergence questions: it is indicated that the "optimal" solution to the real problem to be solved exists, and that the optimal solutions of the Stochastic Optimization Model converge to this solution if the number of realizations increases.

The model has been tested on instances derived from the timetable of NS Reizigers for 2007. These computational experiments show that the robustness of a timetable can be improved by slight modifications of the timetable: the results obtained by the model always provide an improvement over the original timetable, and these results are rather stable under varying intensities of the disturbance distributions.

Moreover, the results of the model were tested in real-life on the timetable of NS Reizigers for 2006 on the "Zaanlijn". There it turned out that the results of the model were quite similar to what was expected: with small disturbances the resulting delays decreased, and with larger disruptions a positive effect could hardly be noticed. The overall effect of the timetable modification was considered as positive.

Note that an increase in the punctuality is quite important for NS Reizigers, since agreements with the government on the license to operate trains on the Dutch railway infrastructure are partly based on punctuality. Moreover, the improvements in the punctuality that can be obtained by applying the Stochastic Optimization Model are nearly for free, whereas other measures to improve the punctuality are likely to be expensive.

The current Stochastic Optimization Model is solved in a straightforward way by applying CPLEX. This restricts both the number of trains in the timetable that can be handled at the same time as well as the number of realizations. However, in our future research we will focus on the development of dedicated optimization techniques for solving the model.

We will also focus on the development of a practical implementation of our model as part of the timetabling system DONS, see Hooghiemstra et al. (1999) and Peeters (2003). This implementation will include e.g. a model for automatically detecting crossing routes of trains in stations. 


\section{References}

Bergmark, R.: Railroad capacity and traffic analysis using SIMON. In: J. Allan, C.A. Brebbia, R.J. Hill, and G. Sciutto (eds.), Computers in Railways V, (1996) 183-191. WIT Press, Ashurst, UK.

Birge, J.R., and F. Louveaux: Introduction to Stochastic Programming, (1997). Springer, New York.

Carey, M.: Ex ante heuristic measures of schedule reliability, Transportation Research B, 33(7) (1999) 473-494.

Ferreira, L., and A. Higgins. Modeling the reliability of train arrival times. Journal of Transportation Engineering, 22(6) (1996) 414-420.

Goverde, R.M.P.: The Max-Plus Algebra approach to railway timetable design. In: B. Mellitt, R.J. Hill, J. Allan, G. Sciutto, and C.A. Brebbia, (eds.), Computers in Railways VI, (1998) 339-350. WIT Press, Ashurst.

Goverde, R.M.P.: Punctuality of railway operations and timetable stability analysis. Ph.D. thesis, Technical University Delft, (2005)

Goverde, R.M.P.: G. Hooghiemstra, H.P. Lopuhaa. Statistical analysis of train traffic: the Eindhoven Case. TRAIL Studies in Transportation Science, S2001/1, (2001), TRAIL Research School, Delft.

Hallowell, S.F., and P.T. Harker: Predicting on-time performance in scheduled railroad operations: methodology and application to train scheduling, Transportation Research A, 32(6) (1998) 279-295.

Higgins, A., and E. Kozan: Modelling train delays in urban networks, Transportation Science, 32(4) (1998) 346-357.

Hooghiemstra, J.S., L.G. Kroon, M.A. Odijk, M. Salomon, and P.J. Zwaneveld: Decision Support Systems support the search for win-win solutions in railway network design. Interfaces, 35 (1999) 15-32.

Hürlimann, D.: Objektorientierte Modellierung von Infrastrukturelementen und Betriebsvorgängen im Eisenbahnwesen (Object oriented modeling of infrastructure elements and business processes in railways). Ph.D. thesis, ETH Zürich, (2001) (in German).

Huisman, T., and R.J. Boucherie: Running times on railway sections with heterogeneous train traffic. Transportation Research B, 35 (2001) 271-292.

Kaniovski, Y.M., A.J. King, R.J.-B. Wets: Probabilistic bounds (via large deviations) for the solutions of Stochastic Programming problems. Annals of Operations Research, 56 (1995), 189-208.

King, A.J., T. Rockafellar. Asymptotic theory for solutions in statistical estimations and Stochastic Programming. Mathematics of Operations Research, 18 (1993) 148162.

Klein Haneveld, W.K., and M.H. van der Vlerk: Stochastic integer programming: general models and algorithms. Annals of Operations Research, 85 (1998) 39-57.

König, H.: VirtuOS: Simulieren von Bahnbetrieb. Betrieb und Verkehr, 50(1-2) (2001) 44-47 (in German).

Kort, A.F. de: Advanced railway planning using Max-Plus algebra. In: J. Allan, C.A. Brebbia, R.J. Hill, and G. Sciutto (eds.), Computers in Railways VII, (2000) 257-266. WIT Press, Ashurst, UK.

Kroon, L.G., R. Dekker, and M.J.C.M. Vromans. Cyclic railway timetabling: a stochastic optimisation approach. To appear in: F. Geraets, L.G. Kroon, A. Schöbel, D. Wagner, and C. Zaroliagis (eds.), Algorithmic Methods in Railway Optimisation. Lecture Notes in Computer Science, Springer, (2007). 
Linderoth, J.T., A. Shapiro, and S.J. Wright: The emprirical behavior of sampling methods for stochastic programming. Optimization Technical Report 02-01. University of Wisconsin-Madison, (2002).

Maróti, G.: A convex optimization approach to robust timetabling. Working paper, Erasmus University Rotterdam, Rotterdam School of Management, (2006).

Middelkoop, D., and M. Bouwman: Train network simulator for support of network wide planning of infrastructure and timetables. In: J. Allan, C.A. Brebbia, R.J. Hill, and G. Sciutto (eds.), Computers in Railways VII, (2000) 267-276. WIT Press, UK.

Nachtigall, K.: Periodic network optimization with different arc frequencies. Discrete Applied Mathematics, 69 (1996) 1-17.

Peeters, L.W.P.: Cyclic railway timetable optimization. Ph.D. thesis, Erasmus University Rotterdam (2003), Rotterdam School of Management.

Petersen E.R., and A.J. Taylor: A structured model for rail line simulation and optimization. Transportation Science 18 (1982) 192-206.

Retel Helmrich, M.J.: Improving robustness of a railway timetable through stochastic optimisation of supplementary times. M.Sc. thesis, Erasmus University Rotterdam, Rotterdam School of Economics, (2006).

Rudoplph, R.: Entwicklung von Strategieen zur optimierten Anordnung und Dimensionierung von Zeitzuschlägen im Eisenbahnbetrieb. Ph.D. thesis, Universität Hannover (2004) (in German).

Schwanhäußer, W.: Die Bemessung der Pufferzeiten im Fahrplangefüge der Eisenbahn. Veröffentlichungen der Verkehrswissenschagtligen Instituts der RWTH Aachen, 20 (1974).

Serafini, P., and W. Ukovich: A mathematical model for Periodic Event Scheduling Problems. SIAM Journal on Discrete Mathematics, 2 (1989) 550-581.

Shapiro, A. Stochastic programming by Monte Carlo simulation methods, Stochastic Programming E-Print Series (2000).

Shapiro, A., T. Homem-de-Mello. On the rate of convergence of optimal solutions of Monte Carlo approximations of stochastic programs. SIAM Journal on Optimization, 11(1) (2000) 70-86.

Soto y Koelemeijer, G., A.R. Iounoussov, R.M.P. Goverde, and R.J. van Egmond: PETER, a performance evaluator for railway timetables. In: J. Allan, C.A. Brebbia, R.J. Hill, and G. Sciutto (eds.), Computers in Railways VII, (2000) 405-414. WIT Press, Ashurst, UK.

U.I.C.: Timetable recovery margins to guarantee timekeeping - Recovery margins, Leaflet 451-1, (2000) U.I.C., Paris, France.

Vromans, M.J.C.M.: Reliability of railway systems. Ph.D. thesis, Erasmus University Rotterdam, Rotterdam School of Management, (2005).

Wahlborg, M.: Simulation models: important aids for Banverket's planning process. In: J. Allan, C.A. Brebbia, R.J. Hill, and G. Sciutto (eds.), Computers in Railways V, (1996) 175-181. WIT Press, Ashurst, UK.

Wakob, H. (1985). Ableitung eines generellen Wartemodells zur Ermittlung der planmässigen Wartezeiten im Eisenbahnbetrieb unter besonderer Berücksichtigung der Aspekte Leistungsfähigkeit und Anlagebelastung. Ph.D. thesis, Verkehrwissenschaftlichen Institut, RWTH Aachen (1985) (in German).

Weigand, W.: Verspätungsübertragungen in Fernverkehrsnetzen. Eisenbahntechnische Rundschau, 30 (1981) 915-920 (in German).

Yuan, J.: Stochastic modelling of train delays and delay propagation in stations. Ph.D. thesis, Technical University Delft, (2006). 


\section{Publications in the Report Series Research ${ }^{*}$ in Management}

\section{ERIM Research Program: "Business Processes, Logistics and Information Systems"}

2006

Smart Business Networks Design and Business Genetics

L-F Pau

ERS-2006-002-LIS

http://hdl.handle.net/1765/7319

Designing and Evaluating Sustainable Logistics Networks

J. Quariguasi Frota Neto, J.M. Bloemhof-Ruwaard, J.A.E.E. van Nunen and H.W.G.M. van Heck ERS-2006-003-LIS

http://hdl.handle.net/1765/7320

Design and Control of Warehouse Order Picking: a literature review

René de Koster, Tho Le-Duc and Kees Jan Roodbergen

ERS-2006-005-LIS

http://hdl.handle.net/1765/7322

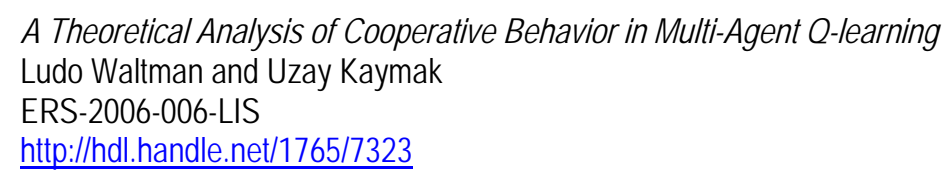

Supply-Chain Culture Clashes in Europe. Pitfalls in Japanese Service Operations M.B.M. de Koster and M. Shinohara

ERS-2006-007-LIS

http://hdl.handle.net/1765/7330

From Discrete-Time Models to Continuous-Time, Asynchronous Models of Financial Markets Katalin Boer, Uzay Kaymak and Jaap Spiering

ERS-2006-009-LIS

http://hdl.handle.net/1765/7546

Mobile Payments in the Netherlands: Adoption Bottlenecks and Opportunities, or... Throw Out Your Wallets Farhat Shaista Waris, Fatma Maqsoom Mubarik and L-F Pau

ERS-2006-012-LIS

http://hdl.handle.net/1765/7593

Hybrid Meta-Heuristics for Robust Scheduling M. Surico, U. Kaymak, D. Naso and R. Dekker ERS-2006-018-LIS

http://hdl.handle.net/1765/7644

VOS: A New Method for Visualizing Similarities between Objects

Nees Jan van Eck and Ludo Waltman

ERS-2006-020-LIS

http://hdl.handle.net/1765/7654

On Noncooperative Games, Minimax Theorems and Equilibrium Problems

J.B.G. Frenk and G. Kassay

ERS-2006-022-LIS

http://hdl.handle.net/1765/7809 
An Integrated Approach to Single-Leg Airline Revenue Management: The Role of Robust Optimization S. Ilker Birbil, J.B.G. Frenk, Joaquim A.S. Gromicho and Shuzhong Zhang ERS-2006-023-LIS

http://hdll.handle.net/1765/7808

Optimal Storage Rack Design for a 3D Compact AS/RS with Full Turnover-Based Storage

Yu Yugang and M.B.M. de Koster

ERS-2006-026-LIS

http://hdl.handle.net/1765/7831

Optimal Storage Rack Design for a 3-dimensional Compact AS/RS

Tho Le-Duc, M.B.M. de Koster and Yu Yugang

ERS-2006-027-LIS

http://hdl.handle.net/1765/7839

E-Fulfillment and Multi-Channel Distribution - A Review

Niels Agatz, Moritz Fleischmann and Jo van Nunen

ERS-2006-042-LIS

http://hdl.handle.net/1765/7901

Leveraging Offshore IT Outsoutcing by SMEs through Online Marketplaces

Uladzimir Radkevitch, Eric van Heck and Otto Koppius

ERS-2006-045-LIS

http://hdl.handle.net/1765/7902

Buyer Commitment and Opportunism in the Online Market for IT Services

Uladzimir Radkevitch, Eric van Heck and Otto Koppius

ERS-2006-046-LIS

http://hdl.handle.net/1765/7903

Managing Supplier Involvement in New Product Development: A Multiple-Case Study

Ferrie E.A. van Echtelt, Finn Wynstra, Arjan J. van Weele and Geert Duysters

ERS-2006-047-LIS

http://hdl.handle.net/1765/7949

The Multi-Location Transshipment Problem with Positive Replenishment Lead Times

Yeming Gong and Enver Yucesan

ERS-2006-048-LIS

http://hdll.handle.net/1765/7947

Solving Lotsizing Problems on Parallel Identical Machines Using Symmetry Breaking Constraints

Raf Jans

ERS-2006-051-LIS

http://hdl.handle.net/1765/7985

Urban Distribution: The Impacts of Different Governmental Time-Window Schemes

H.J. Quak and M.B.M. de Koster

ERS-2006-053-LIS

http://hdl.handle.net/1765/8020

Leader-follower Game in VMI System with Limited Production Capacity Considering Wholesale and Retail Prices Yu Yugang, Liang Liang, and George Q. Huang

ERS-2006-054-LIS

http://hdl.handle.net/1765/8194

Privacy Management Service Contracts as a New Business Opportunity for Operators

L-F Pau

ERS-2006-060-LIS

http://hdl.handle.net/1765/8110 
Performance Evaluation of Real-time Scheduling Approaches in Vehicle-based Internal Transport Systems Tuan Le-Anh, M.B.M de Koster and Yu Yugang

ERS-2006-063-LIS

http://hdl.handle.net/1765/8129

Stochastic Improvement of Cyclic Railway Timetables

Leo Kroon, Rommert Dekker, Gábor Maróti, Mathijn Retel Helmrich and Michiel Vromans

ERS-2006-067-LIS

Shunting of Passenger Train Units: an Integrated Approach

Leo Kroon, Ramon M. Lentink and Alexander Schrijver

ERS-2006-068-LIS

* A complete overview of the ERIM Report Series Research in Management: https://ep.eur.nl/handle/1765/1

ERIM Research Programs:

LIS Business Processes, Logistics and Information Systems

ORG Organizing for Performance

MKT Marketing

F\&A Finance and Accounting

STR Strategy and Entrepreneurship 